\title{
Oncogenic miR-96-5p inhibits apoptosis by targeting the caspase-9 gene in hepatocellular carcinoma
}

\author{
NAOTO IWAI, KOHICHIROH YASUI, AKIRA TOMIE, YASUYUKI GEN, KEI TERASAKI, TOMOKO KITAICHI, \\ TOMOHIRO SODA, NOBUHISA YAMADA, OSAMU DOHI, YUYA SEKO, ATSUSHI UMEMURA, \\ TAICHIRO NISHIKAWA, KANJI YAMAGUCHI, MICHIHISA MORIGUCHI, \\ HIDEYUKI KONISHI, YUJI NAITO and YOSHITO ITOH
}

\begin{abstract}
Department of Molecular Gastroenterology and Hepatology, Graduate School of Medical Science,
\end{abstract} Kyoto Prefectural University of Medicine, Kyoto 602-8566, Japan

Received September 21, 2017; Accepted February 9, 2018

DOI: 10.3892/ijo.2018.4369

\begin{abstract}
The aberrant expression or alteration of microRNAs (miRNAs/miRs) contributes to the development and progression of cancer. In the present study, the functions of miR-96-5p in hepatocellular carcinoma (HCC) were investigated. It was identified that miR-96-5p expression was significantly upregulated in primary HCC tumors compared with their nontumorous counterparts. A copy number gain was frequently observed at chromosomal region $7 \mathrm{q} 32.2$ in which the MIR96 locus is located, suggesting that gene amplification may be one of the mechanisms by which miR-96-5p expression is increased in HCC. Transfection of miR-96-5p mimic into HCC cells decreased the expression of CASP9, which encodes caspase-9, the essential initiator caspase in the mitochondrial apoptotic pathway, at the mRNA and protein levels. A putative binding site for miR-96-5p was identified in the CASP 9 3 -untranslated region, and the results of a luciferase assay indicated that $C A S P 9$ is a potential direct target of miR-96-5p. The miR-96-5p mimic increased resistance to doxorubicinand ultraviolet-induced apoptosis through the decrease in caspase-9 expression in HCC cells. Transfection of miR-96-5p inhibitor enhanced the cytotoxic effect of doxorubicin by increasing caspase- 9 expression in the HCC cells, suggesting a synergistic effect between the miR-96-5p inhibitor and doxorubicin. In conclusion, the results of the present study suggest that miR-96-5p, which is frequently upregulated in HCC, inhibits apoptosis by targeting CASP9. Therefore, miR-96-5p may be a potential therapeutic target for HCC.
\end{abstract}

Correspondence to: Dr Kohichiroh Yasui, Department of Molecular Gastroenterology and Hepatology, Graduate School of Medical Science, Kyoto Prefectural University of Medicine, 465 Kajii-cho, Kamigyo-ku, Kyoto 602-8566, Japan

E-mail: yasuik@koto.kpu-m.ac.jp

Key words: microRNA-96-5p, hepatocellular carcinoma, caspase-9, apoptosis, forkhead box O1

\section{Introduction}

Liver cancer is estimated to lead to $\sim 750,000$ mortalities annually worldwide (1). It is more prevalent in men than in women, and is the second leading cause of cancer mortality in men. The most common primary liver cancer is hepatocellular carcinoma (HCC). The risk factors for HCC include infection with hepatitis $\mathrm{B}$ or hepatitis $\mathrm{C}$ virus, alcohol consumption, and non-alcoholic steatohepatitis (2).

MicroRNAs (miRNAs/miRs) are small non-coding RNAs of between 18 and 25 nucleotides in length that serve a crucial function in gene regulation. miRNAs are known to repress thousands of target genes and co-ordinate normal processes, including cellular proliferation, differentiation and apoptosis (3-5). The aberrant expression or alteration of miRNAs contributes to a range of human pathologies, including cancer $(6,7)$. HCC is no exception, and various HCC-specific miRNA signatures have been reported (8). However, the function of miRNAs in the pathogenesis of $\mathrm{HCC}$ remains poorly understood.

One of the hallmarks of human cancer is intrinsic or acquired resistance to apoptosis (9). The evasion of apoptosis contributes to the development and progression of cancer, and to resistance to treatment. Therefore, a better understanding of the molecular mechanisms underlying the resistance of tumors to apoptosis is expected to provide a basis for a rational approach in the development of molecular targeted therapies.

Caspase-9 is the essential initiator caspase required in the mitochondrial apoptotic pathway (10). Upon exposure to intracellular apoptotic stimuli, mitochondria release cytochrome $c$, which binds to an adaptor protein known as apoptotic proteaseactivating factor 1 (Apaf-1); this binding causes Apaf-1 to oligomerize into a wheel-like heptamer called an apoptosome. Apaf-1 in the apoptosome then recruits caspase-9, which is activated in the apoptosome. The activated caspase- 9 is then able to activate downstream executioner caspases, including caspase- $3,-6$ and -7 , and trigger a cascade of events leading to apoptosis. As such, caspase-9 is considered to be a tumor suppressor (11).

To investigate the miRNAs involved in HCC, miRNAs that were upregulated in primary $\mathrm{HCC}$ tumors when compared with non-tumor tissues were screened for using a miRNA 
microarray. The array analysis revealed that miR-96-5p was among the most significantly upregulated miRNAs in HCC tumors (K. Yasui, unpublished data).

In the present study, the functions of miR-96-5p were investigated in HCC. In addition to the forkhead box O1 (FOXOI) gene, which has been identified to be a direct target of miR-96-5p (12-16), the caspase-9 gene (CASP9) gene was identified as a potential novel target of miR-96-5p. These results suggest that increased miR-96-5p expression may contribute to resistance to apoptosis via the repression of caspase-9 expression in $\mathrm{HCC}$.

\section{Materials and methods}

Reagents and antibodies. Antibodies against caspase-9 (cat. no. 9508), caspase-2 (cat. no. 2224), FOXO1 (cat. no. 2880) and poly(ADP-ribose) polymerase (PARP; cat. no. 9542) were purchased from Cell Signaling Technology, Inc. (Danvers, MA, USA). The antibody against $\beta$-actin (cat. no. A1978) was purchased from Sigma-Aldrich; Merck KGaA (Darmstadt, Germany). Doxorubicin was obtained from Toronto Research Chemicals Inc. (Toronto, ON, Canada).

Cell culture and primary tumor samples. Three HCC cell lines (SNU387, SNU449 and HLF) were obtained from the American Type Culture Collection (Manassas, VA, USA) or the Japan Collection of Research Bioresources Cell Bank (Osaka, Japan). All cell lines were cultured in Dulbecco's modified Eagle's medium containing $10 \%$ fetal bovine serum at $37^{\circ} \mathrm{C}$. Primary $\mathrm{HCC}$ tumor samples and non-tumor samples were obtained from patients who had undergone surgical resection of tumors, as described previously (17). The present study, including the collection and analytical methods, was approved by the Ethics Committees of the Kyoto Prefectural University of Medicine and was conducted in accordance with the Declaration of Helsinki. Written informed consent was obtained from each patient for the use of their tissue samples in the present study.

Reverse transcription-quantitative polymerase chain reaction (RT-qPCR) of miRNA and mRNA. Total RNA was obtained using TRIzol ${ }^{\circledast}$ (Thermo Fisher Scientific, Inc., Waltham, MA, USA). Residual genomic DNA was removed by incubating the RNA samples with RNase-free DNase I (Takara Bio, Inc., Otsu, Japan) prior to performing RT-qPCR.

Quantification of human (hsa)-miR-96-5p was performed using a fluorescence detection method. Single-stranded cDNA was generated using a Universal cDNA synthesis kit II (Exiqon A/S, Vedbaek, Denmark), according to the manufacturer's protocol. RT-qPCR experiments were performed using the StepOnePlus PCR system (Applied Biosystems; Thermo Fisher Scientific, Inc.) with ExiLENT SYBR-Green master mix (Exiqon A/S). The hsa-miR-96-5p locked nucleic acid (LNA) PCR primer set was purchased from Exiqon A/S. Small nucleolar RNA U49 (Exiqon A/S) was used as an endogenous control for miRNA levels.

The mRNAs were detected by RT-qPCR as described previously (18). The expression level of $\beta$-actin was used as an endogenous control for mRNA levels. The primer sequences used for PCR are presented in Table I.
Single-nucleotide polymorphism (SNP) array analysis. Genomic DNA was isolated as described previously (18). Changes in DNA copy number were analyzed in 34 primary HCC tumors using the GeneChip Mapping 250K Sty array (Affymetrix; Thermo Fisher Scientific, Inc.) according to the manufacturer's protocol and as described previously $(18,19)$.

Immunoblotting. Immunoblotting was performed as described previously (18). The dilutions of the antibodies were 1:1,000 for anti-caspase-9, anti-caspase-2, anti-FOXO1 and anti-PARP; and 1:5,000 for $\beta$-actin. For immunodetection, anti-rabbit IgG or anti-mouse IgG (Cell Signaling Technology, Inc.) was used as the secondary antibody at a dilution of 1:5,000 or 1:10,000, respectively. Antibody binding was detected using the enhanced chemiluminescence system (GE Healthcare, Chicago, IL, USA).

Transfection of miRNA mimics and inhibitors. Hsa-miR-96-5p mimic (\#472140-001), cel-miR-39-3p (\#479902-001), which was used as a miRNA mimic negative control, hsa-miR-96-5p inhibitor (\#4102571-101) and miRNA inhibitor negative control (\#199006-101) were obtained from Exiqon A/S. These mimics and inhibitors were transfected into cells using Lipofectamine ${ }^{\circledR}$ RNAiMAX (Invitrogen; Thermo Fisher Scientific, Inc.), and were used at a final concentration of $12.5 \mathrm{nmol} / 1$, except for SNU449 cells, which were transfected with $12.5,25$ and $50 \mathrm{nmol} / 1 \mathrm{miR}-96-5 \mathrm{p}$ inhibitor. After $72 \mathrm{~h}$ of incubation, cells were harvested for RT-qPCR and immunoblotting analyses.

Identification of the target genes of miR-96-5p. A total of 15 candidate genes were selected (Table II) that were predicted to be target genes of miR-96-5p by miRBase (www.mirbase.org), miRTarBase (mirtarbase.mbc.nctu.edu.tw) and microRNA. org (www.microrna.org). miR-96-5p mimic or negative control mimic was transfected into SNU387 and HLF cells that exhibited low expression levels of miR-96-5p, and the mRNA and protein levels of the 15 genes were determined using RT-qPCR and immunoblotting, respectively, as aforementioned.

Luciferase reporter assay. The 3'-untranslated region (3'-UTR) sequence of CASP9 mRNA, inclusive of the miR-96-5p target site, was cloned downstream of the firefly luciferase gene in the pMirTarget vector (OriGene Technologies, Inc., Rockville, MD, USA). Site-specific mutations were generated in the CASP9 3'-UTR using the KOD Plus Mutagenesis kit (Toyobo Life Science, Osaka, Japan), according to the manufacturer's protocol. The luciferase-CASP9 3'-UTR wild-type (WT) vector, the luciferase-CASP9 3'-UTR mutant vector or empty vector was transfected into SUN387 cells together with either miR-96-5p mimic or negative control mimic using Lipofectamine $^{\circledast} 3000$ (Invitrogen). After $48 \mathrm{~h}$ of incubation, cells were harvested for the measurement of firefly and Renilla luciferase activity using the Dual-Glo Luciferase assay system (Promega Corporation, Madison, WI, USA).

RNA interference. Two small interfering RNAs (siRNAs) targeting CASP9 (\#1 and \#2; Ambion; Thermo Fisher Scientific, Inc.) and control (non-silencing) siRNA were delivered into cells using Lipofectamine RNAiMAX (Invitrogen). After $72 \mathrm{~h}$ of incubation, cells were harvested for immunoblotting. 
Table I. Primer sequences used for PCR.

\begin{tabular}{|c|c|c|}
\hline Gene & Forward primer & Reverse primer \\
\hline FOXO1 & 5'-AAGAGCGTGCCCTACTTCAA-3' & 5'-CTGTTGTTGTCCATGGATGC-3' \\
\hline CASP9 & 5'-GCAAGCAGCAAAGTTGTCGA-3' & 5'-GGACTCACGGCAGAAGTTCA-3' \\
\hline CASP2 & 5'-TGATGCCTTCTGTGAAGCAC-3' & 5'-GCTCAACGGTGGGAGTACAT-3' \\
\hline$C D K N 1 A$ & 5'-ATGAAATTCACCCССТTTCC-3' & 5'-CCCTAGGCTGTGCTCACTTC-3' \\
\hline FOXO3 & 5'-GGCGGACTTTGTGTTTGTTT-3' & 5'-AAGCCACCTGAAATCACACC-3' \\
\hline REVI & 5'-TTGTGATGAAGCGCTGGTAG-3' & 5'-TTGGTCACTAGCTGGCCTCT-3' \\
\hline RAD51 & 5'-CGACTCTCCCTGTCTTCCTG-3' & 5'-TTTCCCGGAAGCTTTATCCT-3' \\
\hline BRMS1L & 5'-AGTGAAAACGGAACCACCTG-3' & 5'-CCATCAGGCCTCTTAAACCA-3' \\
\hline EFNA5 & 5'-AAACGTATTGTGCCCTGGAG-3' & 5'-CTGCCACTCCAGAGGAGTTC-3' \\
\hline AKT1S1 & 5'-AGGGCTCTTTGTGATGGATG-3' & 5'-ACTTGGCGTACTGCTGTGTG-3' \\
\hline$H B P 1$ & 5'-CCGTGAAAATGAGGTGGACT-3' & 5'-GAAGGCTGGTTCACTCTTCG-3' \\
\hline PAX6 & 5'-CCGGCAGAAGATTGTAGAGC-3' & 5'-CTCACACATCCGTTGGACAC-3' \\
\hline IRS1 & 5'-GCTTTCCACAGCTCACCTTC-3' & 5'-CCTCAGTGCCAGTCTCTTCC-3' \\
\hline STK17A & 5'-TTTGTCTGAGTCGGCTGTTG-3' & 5'-GTGCCTTTTCCATCCTGAAA-3' \\
\hline PLAGL1/ZAC & 5'-CAAGTGTGTGCAGCCTGACT-3' & 5'-ACACTCCTCACACCCAAAGG-3' \\
\hline$A C T B$ & 5'-GTCCACCTTCCAGCAGATGT-3' & 5'-TGTTTTCTGCGCAAGTTAGG-3' \\
\hline
\end{tabular}

Table II. Candidate target genes of miR-96-5p.

\begin{tabular}{lcc}
\hline & \multicolumn{2}{c}{ Expression ratio } \\
\cline { 2 - 3 } Gene & SNU387 & HLF \\
\hline FOXO1 & 0.30 & 0.44 \\
CASP9 & 0.37 & 0.25 \\
CASP2 & 0.53 & 0.44 \\
CDKN1A & 0.79 & 0.64 \\
FOXO3 & 0.78 & 0.75 \\
REV1 & 0.55 & 0.55 \\
RAD51 & 0.80 & 0.65 \\
BRMS1L & 0.53 & 0.50 \\
EFNA5 & 1.35 & 1.67 \\
AKT1S1 & 0.61 & 0.84 \\
HBP1 & 0.42 & 0.38 \\
PAX6 & 1.40 & 0.55 \\
IRS1 & 0.59 & 0.94 \\
STK17A & 0.33 & 0.24 \\
PLAGL1/ZAC & 0.60 & 0.64 \\
\hline
\end{tabular}

Gene expression levels were determined using RT-qPCR in SNU387 and HLF cells that were transfected with miR-96-5p mimic or control mimic. Values were normalized to levels in cells transfected with control mimic.

Cell viability assay. Cell viability was determined using the water-soluble tetrazolium salt 8 assay (Cell Counting Kit-8; Dojindo Molecular Technologies, Inc., Kumamoto, Japan), according to the manufacturer's protocol.

Apoptosis assay. Cells were transfected with miR-96-5p mimic or negative control mimic. After $48 \mathrm{~h}$ of incubation, the cells were treated with doxorubicin or exposed to ultraviolet (UV) irradiation at $40 \mathrm{~J} / \mathrm{m}^{2}$ (SNU387 cells) or $10 \mathrm{~J} / \mathrm{m}^{2}$ (HLF cells) using a UV HybriLinker HL-2000 (BM Equipment Co., Ltd., Tokyo, Japan). Apoptosis was evaluated from the expression level of cleaved PARP by immunoblotting and the levels of caspase-3/7 activities using the Caspase-Glo 3/7 assay (Promega Corporation).

Statistical analysis. Statistical analyses were performed using SPSS Statistics (version 24.0; IBM Corp., Armonk, NY, USA). Comparisons were made using the Wilcoxon signed-rank test, Mann-Whitney U test, Student's t-test or analysis of variance (ANOVA) followed by post hoc tests. $\mathrm{P}<0.05$ was considered to indicate a statistically significant difference.

\section{Results}

Expression of miR-96-5p is upregulated in primary $H C C$ tumors. Using RT-qPCR to analyze 27 paired tumor and nontumor tissues, it was identified that miR-96-5p expression was significantly upregulated in the HCC tumors compared with their non-tumorous counterparts (Fig. 1A). To investigate the molecular mechanisms by which miR-96-5p is upregulated in the HCC tumors, aberrations in DNA copy number were examined in primary HCC tumors. High-resolution SNP array analysis revealed a frequent copy number gain at chromosomal region 7q32.2, where the MIR96 locus is located; it was identified in 17/34 (50\%) HCC tumors (Fig. 1B). The presence of a copy number gain at the MIR96 locus was significantly associated with increased expression levels of miR-96-5p in the HCC tumors (Mann-Whitney U test; Fig. 1C).

The potential association between the increased expression levels of miR-96-5p in HCC tumors and various clinicopathological parameters was analyzed. However, no significant association was apparent between the levels of miR-96-5p and any of the parameters, including age, sex, tumor size, 
A

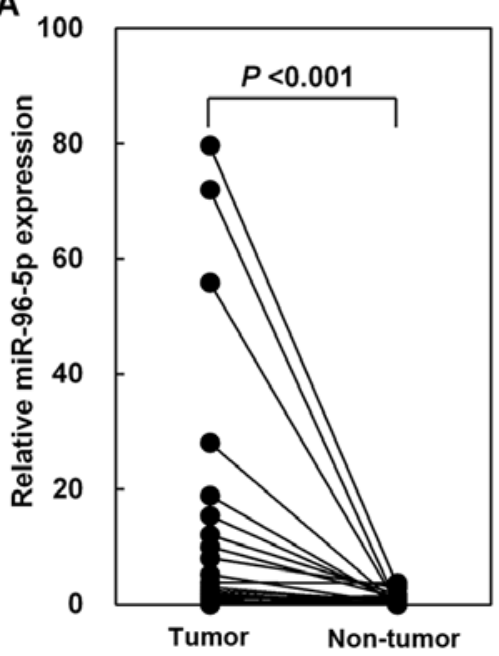

B

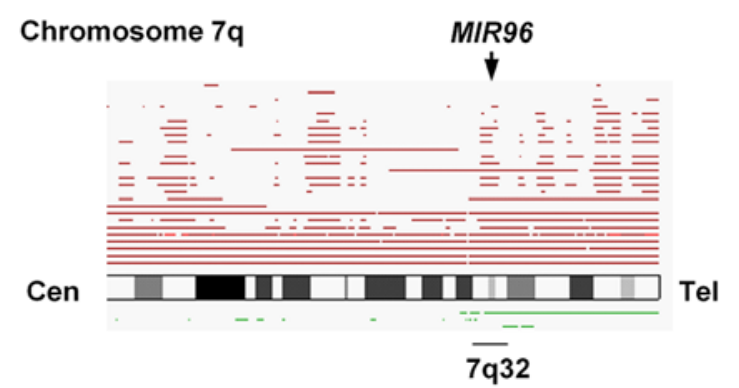

C

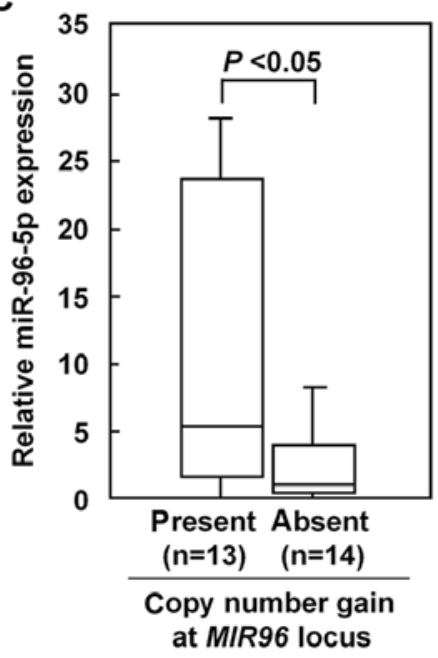

Figure 1. (A) Relative expression levels of miR-96-5p in paired tumor and non-tumor liver tissues from 27 patients with primary HCC were determined using reverse transcription-quantitative polymerase chain reaction. (B) Recurrent copy number gains in the chromosomal region $7 \mathrm{q} 32$ were assessed in primary HCC tumors using a GeneChip Mapping 250K Sty array. Copy number gains are indicated by red horizontal lines above the chromosome ideogram. Copy number losses are indicated by green lines under the chromosome ideogram. Each horizontal line represents an aberration detected in a single HCC tumor. The position of the MIR96 locus is indicated by a black arrow. (C) Association between a copy number gain at the MIR96 locus and the expression levels of miR-96-5p in primary HCC tumors. The horizontal lines represent the means. The boxes contain the values between the 25 th and 75 th percentiles. The error bars denote the 5th and 95th percentile values. HCC, hepatocellular carcinoma; MIR96, microRNA-96; Cen, centromere; Tel, telomere.

clinical stage, hepatitis $\mathrm{B}$ or hepatitis $\mathrm{C}$ virus infection and $\alpha$-fetoprotein level. No association was observed between the levels of miR-96-5p and overall or disease-free survival (data not shown).

CASP9 is identified as a novel direct target of miR-96-5p. To identify the target genes of miR-96-5p, it was investigated whether or not enforced expression of miR-96-5p was able to decrease the expression levels of the 15 candidate genes. These genes were selected because they were predicted to be target genes of miR-96-5p using an in silico approach, and they were potential tumor suppressor genes. Results of the RT-qPCR analysis are presented in Table II and Fig. 2A. Representative images of immunoblotting are presented in Fig. 2B. Of the 15 candidate genes, FOXO1, CASP 9 and $C A S P 2$ exhibited decreased expression at the mRNA and protein levels following transfection with the miR-96-5p mimic compared with transfection with the control mimic in SNU387 and HLF cells (Fig. 2A and B). To confirm these results, rescue experiments were performed using the miR-96-5p inhibitor in combination with the miR-96-5p mimic. Immunoblot analyses revealed that transfection with the miR-96-5p inhibitor attenuated the suppression of FOXO1, caspase- 9 and caspase- 2 protein expression by the miR-96-5p mimic (Fig. 2C).

A luciferase assay was performed to investigate whether or not $C A S P 9$ is a direct target of miR-96-5p using a $C A S P 9$ 3'-UTR-luciferase reporter construct. A putative binding site of miR-96-5p was identified in the CASP9 3'-UTR (Fig. 2D). SNU387 cells that were co-transfected with the luciferase-CASP9 3'-UTR WT vector and miR-96-5p mimic exhibited significantly decreased luciferase activity compared with the cells co-transfected with the luciferase-CASP9 3'-UTR WT vector and control mimic (Fig. 2D). In addition, no decrease in luciferase activity was observed when the empty luciferase reporter vector or the luciferase-CASP9 3'-UTR mutant vector was co-transfected with the miR-96-5p mimic (Fig. 2D). Regarding CASP2, the luciferase assay was not able to demonstrate that $C A S P 2$ was a direct target of miR-96-5p (data not shown).

Furthermore, the mRNA levels of FOXO1 and CASP9 were determined using RT-qPCR in paired tumor and nontumor liver tissues from 35 patients with primary HCC. The mRNA levels of FOXO1 and CASP 9 were decreased in the tumors compared with in their non-tumorous counterparts, although the difference was only statistically significant for FOXO1 (Fig. 2E). However, contrary to expectations, there was no inverse association between the expression levels of miR-96-5p and the mRNA levels of FOXOI or CASP9 in the HCC tumors (data not shown).

miR-96-5p-mediated suppression of caspase-9 inhibits apoptosis. To examine whether miR-96-5p inhibits apoptosis by decreasing caspase- 9 expression, caspase- 9 expression was knocked down in SNU387 and HLF cells by transfection with two different siRNAs targeting CASP9, followed by treatment with doxorubicin, an anticancer agent that causes DNA strand breaks. Caspase-9 knockdown was confirmed by immunoblotting (Fig. 3A). Doxorubicin decreased the cell viability of SNU387 and HLF cells in a dose-dependent manner. However, knockdown of caspase-9 attenuated the doxorubicin-induced decrease in cell viability in SNU387 and HLF cells, when SNU387 cells were treated with doxorubicin at $0.3,1.2$ and $5.0 \mu \mathrm{mol} / 1$ and HLF cells were treated with doxorubicin at 0.15 and $0.3 \mu \mathrm{mol} / 1$ (Fig. 3B).

SNU387 and HLF cells were transfected with the miR-96-5p mimic or control mimic, followed by treatment with doxorubicin. The rate of the doxorubicin-induced decrease in cell viability was significantly lower in cells transfected with the miR-96-5p mimic compared with in cells transfected with the control mimic in a dose-dependent and time-dependent manner (Fig. 3C and D). 
A

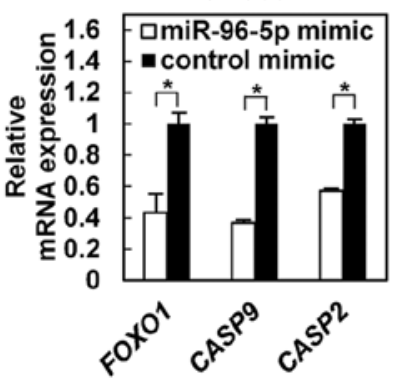

HLF

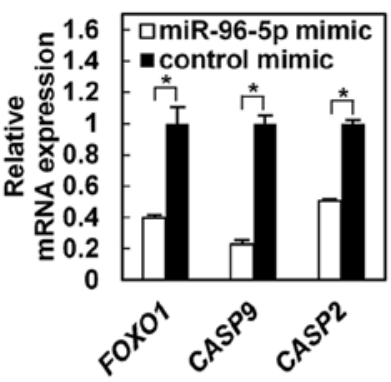

B

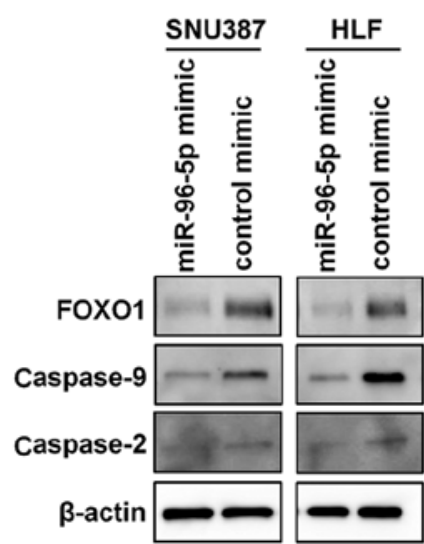

C

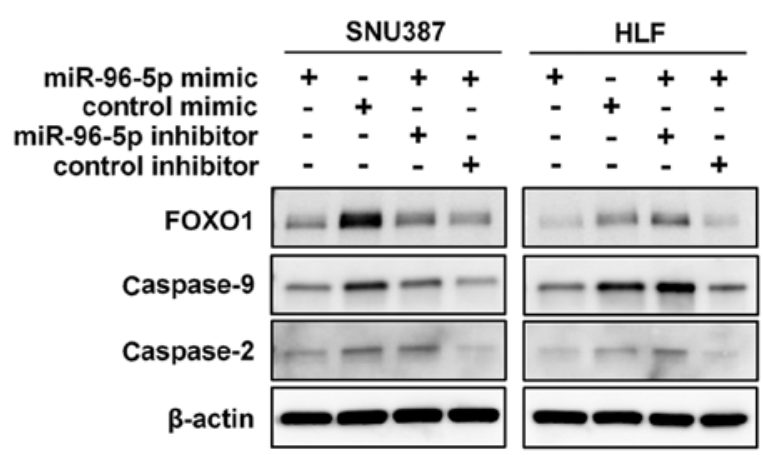

D $\begin{array}{ll}\text { hsa-miR96-5p } & \text { 3'-UCGUUUUUACACGAUCACGGUUU-5' } \\ \text { CASP9 3'-UTR WT } & \text { 5'-CGUCUUCCUGAG- I I I I I I I I } \\ \text { CASP9 3'-UTR mut } & \text { 5'-CGUCUUCCUGAG- UGGACGGAAA-3' }\end{array}$

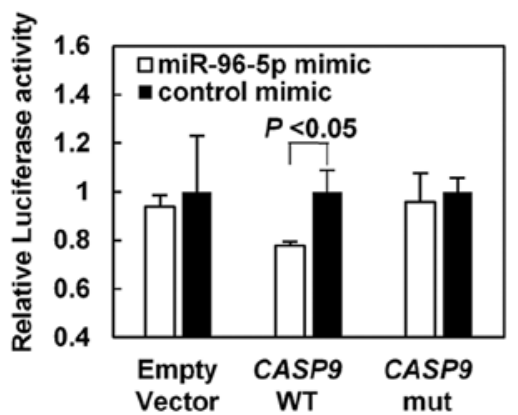

E

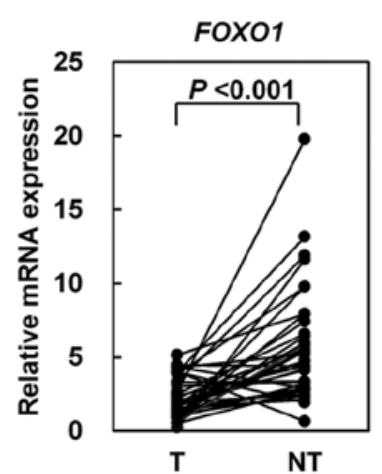

Figure 2. (A) Relative mRNA expression levels of FOXO1, CASP9 and CASP2 in SNU387 and HLF cells transfected with the miR-96-5p mimic or control mimic. (B) Immunoblot analyses of the indicated proteins in SNU387 and HLF cells transfected with the miR-96-5p mimic or control mimic. $\beta$-actin was used as an internal control. (C) Rescue experiments using the miR-96-5p inhibitor in combination with the miR-96-5p mimic. SNU387 and HLF cells were transfected with the miR-96-5p mimic or control mimic in combination with the miR-96-5p inhibitor or control inhibitor. Immunoblot analyses of the FOXO1, caspase-9 and caspase-2 proteins are shown. (D) Upper panel: A putative binding site for miR-96-5p in the CASP9 3'-UTR. Point mutations (*) were introduced by site-directed mutagenesis to destroy the base-paring with the miRNA. Lower panel: Luciferase assay. A luciferase reporter plasmid bearing the CASP9 3'-UTR WT, CASP9 3'-UTR mut or empty vector was co-transfected with the miR-96-5p mimic or control mimic into SNU387 cells. (E) Relative mRNA expression levels of $F O X O 1$ and $C A S P 9$ in paired tumor and non-tumor tissues from 35 patients with primary HCC as determined using reverse transcription-quantitative polymerase chain reaction. FOXO1, forkhead box O1; CASP, caspase; miR, microRNA; UTR, untranslated region; mut, mutant; WT, wild-type; T, tumor; NT, non-tumor.

Finally, SNU387 and HLF cells were transfected with the miR-96-5p mimic or control mimic, followed by treatment with doxorubicin or UV to induce apoptosis. Immunoblotting of caspase-9 and cleaved PARP revealed that, in SNU387 and HLF cells, transfection with the miR-96-5p mimic decreased caspase- 9 expression and attenuated the apoptosis induced by doxorubicin or UV compared with the control mimic (Fig. 3E and F). Results of the caspase-3/7 activity assay indicated that transfection with the miR-96-5p mimic significantly decreased the caspase-3/7 activity in HLF cells treated with doxorubicin compared with similarly treated HLF cells transfected with the control mimic (Fig. 3G).
Apoptosis is induced by the inhibition of miR-96-5p. To evaluate miR-96-5p as a potential therapeutic target for HCC, miR-96-5p inhibitor or control inhibitor was transfected into SNU449 cells, which exhibited increased expression of miR96-5p. SNU449 cells transfected with a high concentration (i.e. $50 \mathrm{nmol} / \mathrm{l}$ ) of miR-96-5p inhibitor exhibited decreased cell viability compared with those transfected with the same concentration of the control inhibitor (Fig. 4A and B). Immunoblotting of cleaved PARP identified that the miR96-5p inhibitor induced apoptosis (Fig. 4C).

Although SNU449 cells transfected with a low concentration (i.e. $10 \mathrm{nmol} / \mathrm{l}$ ) of miR-96-5p inhibitor did not exhibit 
A

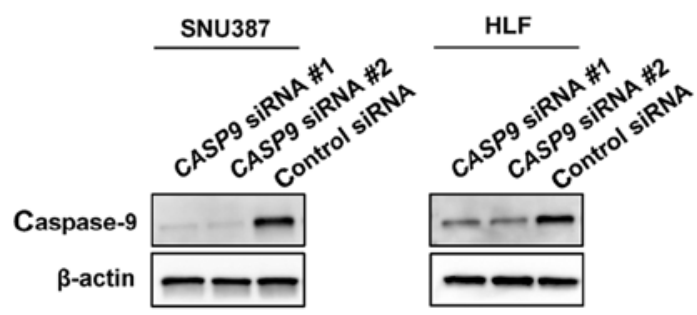

C
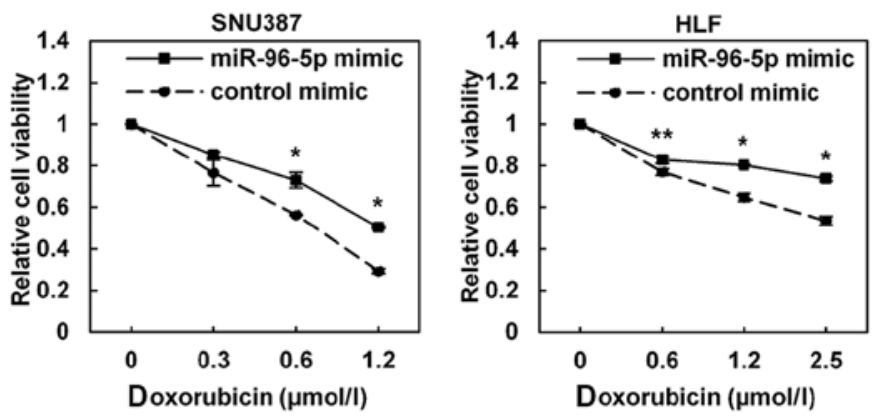

$\mathbf{F}$

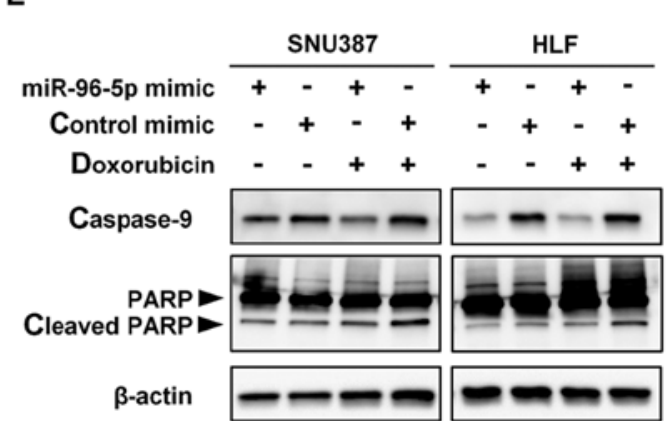

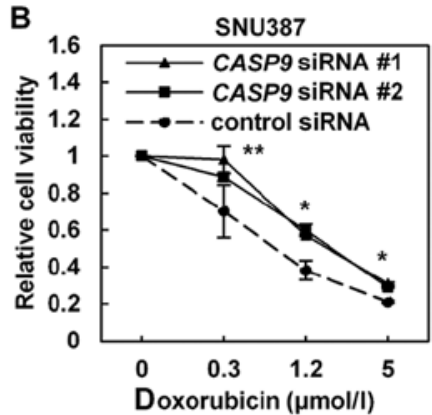

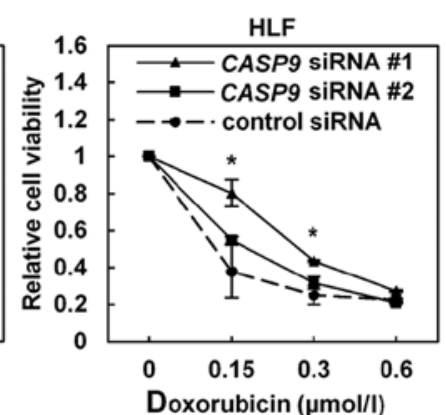

D
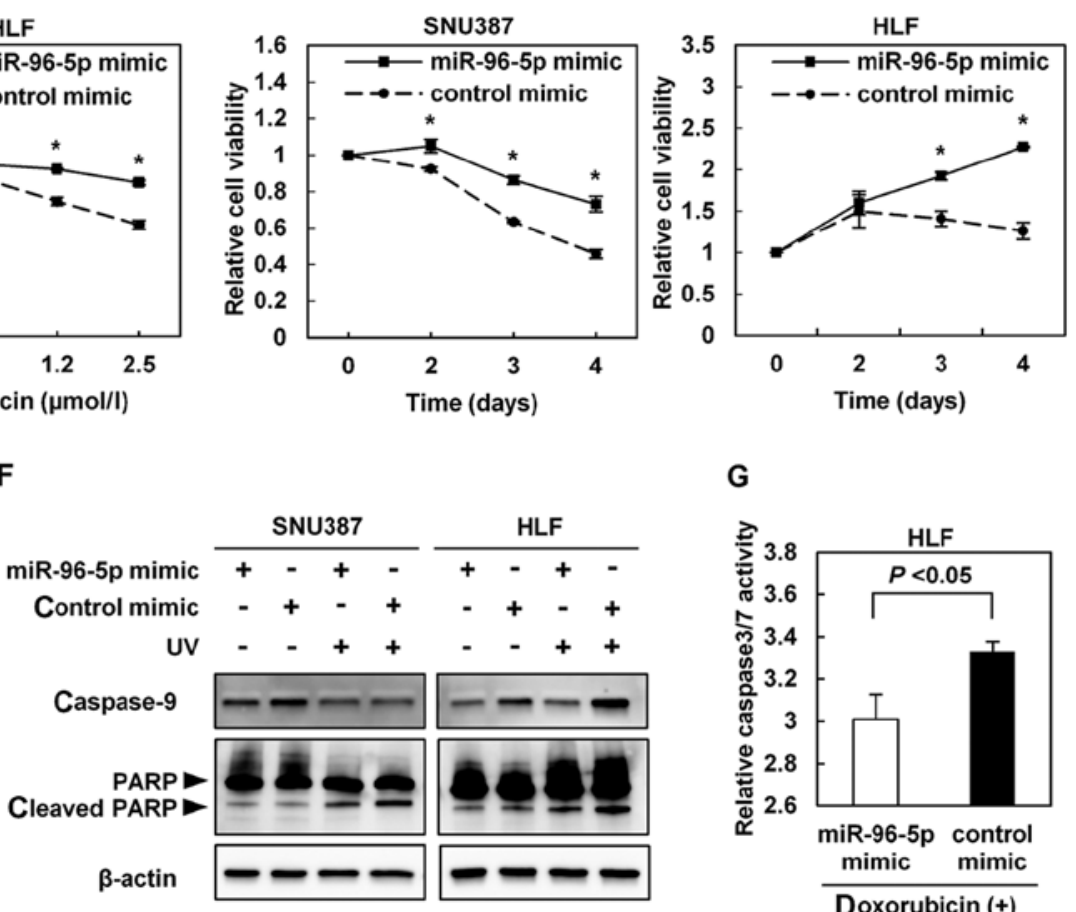

G

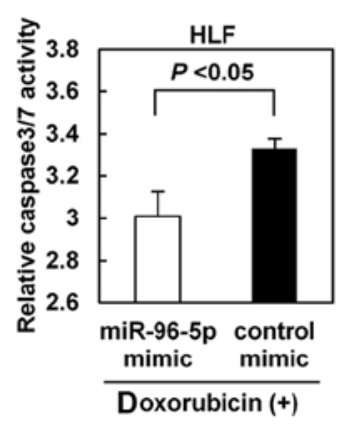

Figure 3. (A) Immunoblot analysis of caspase-9 in SNU387 and HLF cells transfected with CASP9 siRNA (\#1 or \#2) or with control siRNA. (B) Effect of knockdown of caspase-9 on the cytotoxicity of doxorubicin. SNU387 and HLF cells were transfected with CASP9 siRNA (\#1 or \#2) or control siRNA, then treated with doxorubicin $48 \mathrm{~h}$ later at the indicated concentrations. Cell viability was measured $48 \mathrm{~h}$ after doxorubicin treatment. ${ }^{*} \mathrm{P}<0.01$ and ${ }^{* * *} \mathrm{P}<0.05 \mathrm{vs}$. control siRNA. SNU387 and HLF cells were transfected with the miR-96-5p mimic or control mimic, then treated with doxorubicin $48 \mathrm{~h}$ later. (C) SNU387 and HLF cells were treated with doxorubicin at the indicated concentrations. Cell viability was measured $96 \mathrm{~h}$ after doxorubicin treatment. "P<0.01 and ${ }^{* *} \mathrm{P}<0.05$ vs. control mimic. (D) SNU387 and HLF cells were treated with doxorubicin at 0.6 and $1.0 \mu \mathrm{mol} / 1$, respectively. Cell viability was measured at the indicated times. "P<0.01 vs. control mimic. Effect of the miR-96-5p mimic on apoptosis induced by doxorubicin and UV. SNU387 and HLF cells were transfected with the miR-96-5p mimic or control mimic, then treated with doxorubicin or UV $48 \mathrm{~h}$ later. (E) SNU387 cells were treated or not treated with $0.6 \mu \mathrm{mol} / 1$ doxorubicin for $6 \mathrm{~h}$. HLF cells were treated or not treated with $2.5 \mu \mathrm{mol} / 1$ doxorubicin for $12 \mathrm{~h}$. (F) SNU387 cells were exposed or not exposed to UV irradiation at $40 \mathrm{~J} / \mathrm{m}^{2}$. HLF cells were exposed or not exposed to UV irradiation at $10 \mathrm{~J} / \mathrm{m}^{2}$. Subsequently, the cells were cultured for $18 \mathrm{~h}$. Immunoblot analyses of caspase-9, PARP and cleaved PARP as a marker of apoptosis are shown. (G) Measurement of caspase-3/7 activity. HLF cells were transfected with the miR-96-5p mimic or control mimic, then treated with $2.5 \mu \mathrm{mol} / 1$ doxorubicin $48 \mathrm{~h}$ later. Caspase- $3 / 7$ activity was measured $12 \mathrm{~h}$ after doxorubicin treatment. CASP9, caspase-9; siRNA, small interfering RNA; UV, ultraviolet; miR, microRNA; PARP, poly(ADP-ribose) polymerase.

any changes in cell viability (Fig. 4A and D), they did exhibit increased caspase-9 expression, as revealed by immunoblotting (Fig. 4E). In addition, SNU449 cells transfected with $10 \mathrm{nmol} / 1 \mathrm{miR}-96-5 \mathrm{p}$ inhibitor prior to treatment with doxorubicin exhibited significantly lower cell viability compared with the cells transfected with the control inhibitor (Fig. 4F).

\section{Discussion}

In the present study, it was identified that miR-96-5p was significantly upregulated in the HCC tumors compared with in the non-tumor tissues; this result was consistent with the results of previous studies $(13,20)$. miR-96-5p is a putative oncogenic
miRNA that is upregulated in different types of cancer, including breast (14), colorectal (21), prostate (22), bladder (23) and nonsmall cell lung (24) cancer. However, the underlying molecular mechanism of miR-96-5p upregulation in cancer remains unclear. Although previous studies have identified that transforming growth factor- $\beta 1$ (25), hypoxia (26) and mitochondrial dysfunction (27) induced the expression of miR-96-5p, these factors did not increase the expression of miR-96-5p in HCC cells in the present study (data not shown). The reason for this difference is unclear, but it may be due to the different types of cell used. The results of the present study suggest that the copy number gain at the MIR96 locus is one of the molecular mechanisms by which miR-96-5p expression is increased in HCC. 
A

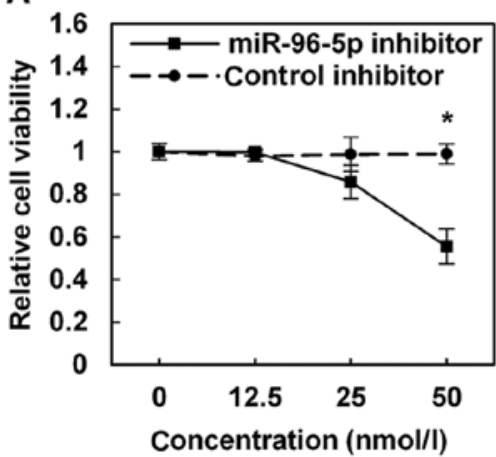

D

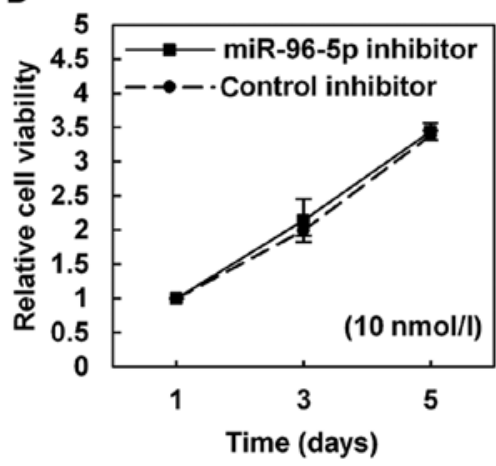

B

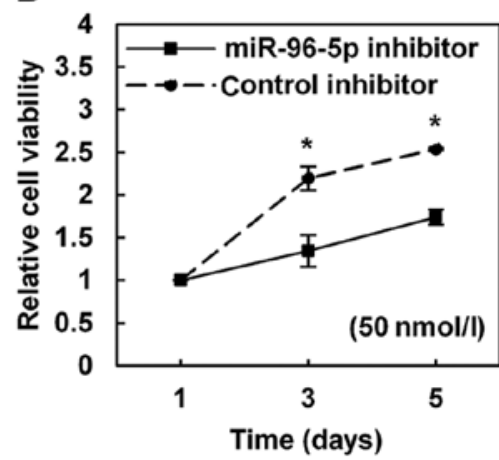

E

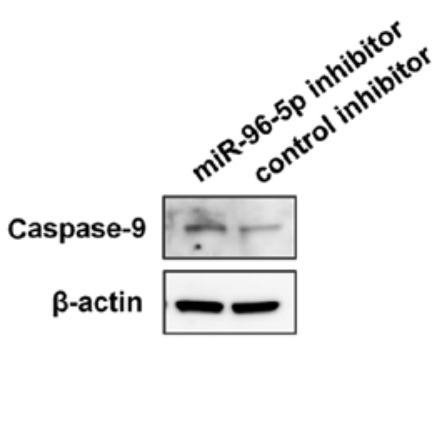

C

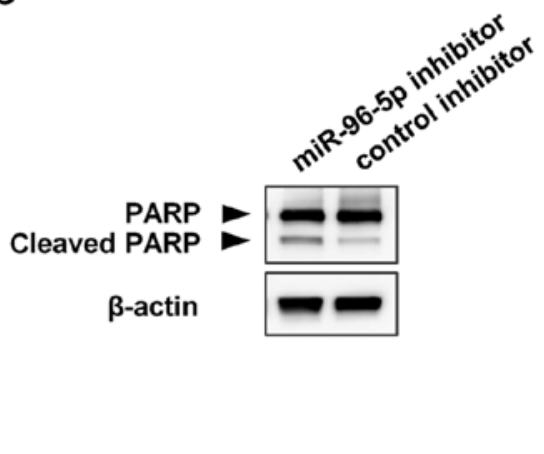

F

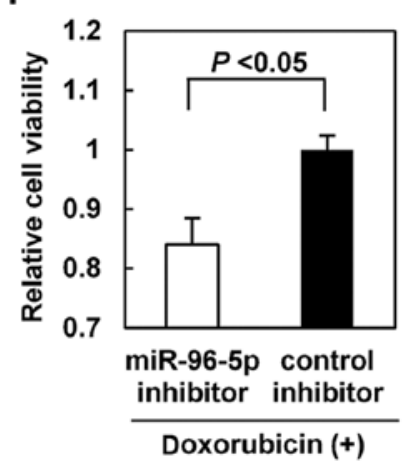

Figure 4. (A) Effect of the miR-96-5p inhibitor on cell viability. SNU449 cells were transfected with the miR-96-5p inhibitor or control inhibitor at the indicated concentrations. Cell viability was assayed 5 days after transfection. ${ }^{*} \mathrm{P}<0.01$ vs. control inhibitor. (B) Effect of a high concentration of miR-96-5p inhibitor on cell viability. SNU449 cells were transfected with $50 \mathrm{nmol} / 1 \mathrm{miR}-96-5 \mathrm{p}$ inhibitor or control inhibitor. Cell viability was assayed at the indicated times. ${ }^{*} \mathrm{P}<0.01$ vs. control inhibitor. (C) Induction of apoptosis by a high concentration of miR-96-5p inhibitor. SNU449 cells were transfected with 50 nmol/1 miR-96-5p inhibitor or control inhibitor, then subjected $48 \mathrm{~h}$ later to immunoblot analysis of cleaved PARP as a marker for apoptosis. (D) Cell viability assay of SNU449 cells transfected with $10 \mathrm{nmol} / 1$ miR-96-5p inhibitor or control inhibitor. (E) Immunoblot analysis of caspase-9 in SNU449 cells transfected with $10 \mathrm{nmol} / 1 \mathrm{miR}-96-5 \mathrm{p}$ inhibitor or control inhibitor. (F) Synergistic effect between the miR-96-5p inhibitor and doxorubicin. SNU449 cells were transfected with $10 \mathrm{nmol} / 1 \mathrm{miR}-96-5 \mathrm{p}$ inhibitor or control inhibitor, then treated with $0.3 \mu \mathrm{mol} / 1$ doxorubicin $48 \mathrm{~h}$ later. Cell viability was assayed $48 \mathrm{~h}$ after doxorubicin treatment. miR, microRNA; PARP, poly(ADP-ribose) polymerase.

miR-96 belongs to the miR-183/-96/-182 cluster, a conserved polycistronic miRNA cluster. The miR-183/-96/-182 cluster is transcribed into the same primary miRNA. Thus, miR-183, miR-96 and miR-182 are likely to have similar expression levels. However, despite the upregulation of miR-96, unpredictably, the microarray and RT-qPCR analyses revealed that miR-183 and miR-182 were not upregulated in the HCC tumors compared with in the non-tumor tissues. Although the reason for this is unknown, it is possible that there is a difference in stability between the three miRNAs in HCC cells.

Next, target genes of miR-96-5p were identified. Each miRNA is predicted to negatively regulate tens to hundreds of target genes. Several previous studies have identified that FOXO1 is a direct target of miR-96-5p in $\operatorname{HCC}(12,13)$, and in other types of malignancy, including breast (14), prostate (15) and endometrial (16) cancer. FOXO1 orchestrates the regulation of genes involved in the apoptotic response, cell cycle checkpoints and cellular metabolism $(14,28)$. FOXO1 is a putative tumor suppressor (29) and the expression of this gene is downregulated in various types of cancer. In addition, it appears that FOXO1-dependent cell cycle arrest and apoptosis are critical for its tumor-suppressive effect. The results of the present study indicated that the miR-96-5p mimic decreased the expression of $F O X O 1$ at the mRNA and protein levels in HCC cells, and that the mRNA levels of FOXO1 were significantly decreased in the primary HCC tumors compared with in their non-tumorous counterparts, confirming that FOXO1 is a target of miR-96-5p in HCC. Although miR-183 and miR-182 were not upregulated in the HCC tumors in the present study, it has been reported that miR-183, miR-96 and miR-182 concordantly downregulated FOXO1 in HCC cells (13). In addition to FOXO1, previous studies have identified that several genes, including those encoding forkhead box O3 (30), glypican 3 (31), insulin receptor substrate 1 (27), reversion inducing cysteine-rich protein with Kazal motifs (32), metastasis suppressor 1 (33) and protein tyrosine phosphatase non-receptor 9 (34), are potential targets of miR-96-5p in cancers.

The present study attempted to identify novel target genes of miR-96-5p in HCC. Using an in silico approach, the putative binding site of miR-96-5p was identified in the CASP9 3'-UTR. RT-qPCR and immunoblot analyses revealed that the miR-96-5p mimic decreased CASP9 expression at the mRNA and protein levels, respectively. Results of the luciferase assay using the CASP9 3'-UTR-luciferase reporter construct suggested that $C A S P 9$ is a potential direct target of miR-96-5p. Furthermore, the mRNA levels of CASP 9 were decreased in the primary HCC tumors compared with in their non-tumorous counterparts. Collectively, the results of the present study suggested that CASP9 is a novel direct target of miR-96-5p, 
and that CASP9 expression is decreased by the upregulation of miR-96-5p in HCC. Since caspase-9 is the essential initiator caspase required for apoptosis signaling and it is considered to be a tumor suppressor, downregulation of caspase- 9 by miR-96-5p may be beneficial to cancers.

Resistance to apoptosis contributes to the development and progression of cancer, and to resistance to chemotherapy. Indeed, the present study demonstrated that knockdown of caspase-9 resulted in resistance to doxorubicin-induced apoptosis. Furthermore, it was identified that enforced expression of miR-96-5p led to resistance to doxorubicin- and UV-induced apoptosis through the downregulation of caspase-9. These results suggested that miR-96-5p inhibits apoptosis by decreasing caspase- 9 expression. Therefore, upregulation of miR-96-5p may be involved in the development and progression of HCC, and may contribute to resistance to chemotherapy by inhibiting apoptosis through a decrease in caspase-9 expression in HCC. Furthermore, because FOXO1 also induces apoptosis, the miR-96-5p-mediated decrease in FOXO1 and caspase-9 expression may have a synergistic effect on inhibiting apoptosis.

Finally, it was investigated whether miR-96-5p may be a potential therapeutic target in HCC. A high concentration (i.e. $50 \mathrm{nmol} / \mathrm{l}$ ) of miR-96-5p inhibitor decreased the viability of HCC cells by inducing apoptosis. Although the molecular mechanism underlying this apoptosis is unclear and the high concentration of the inhibitor used may have caused offtarget effects, these results suggest that miR-96-5p may be a potential therapeutic target. Interestingly, a low concentration (i.e. $10 \mathrm{nmol} / \mathrm{l}$ ) of miR-96-5p inhibitor, which did not affect the viability of the HCC cells, enhanced the cytotoxic effect of doxorubicin by increasing caspase- 9 expression. These results suggested that HCC cells may be sensitized to doxorubicin by pretreatment with a low concentration of miR-96-5p inhibitor, and that there is a synergistic effect between the miR-96-5p inhibitor and doxorubicin. Of note, in previous studies in which small molecules against miR-96-5p were designed, it was demonstrated that the drugs induced apoptosis in breast cancer cells while leaving healthy cells unaffected $(35,36)$. Taken together, these results and those of the present study indicate that miR-96-5p inhibitor, alone or together with a cytotoxic drug, may provide a promising strategy for the treatment of $\mathrm{HCC}$.

In conclusion, the results of the present study suggest that miR-96-5p, which is frequently upregulated in HCC, inhibits apoptosis by targeting CASP9. Therefore, miR-96-5p may be a potential therapeutic target for HCC.

\section{Acknowledgements}

Not applicable.

\section{Funding}

The present study was supported by the Japan Society for the Promotion of Science KAKENHI (grant no. 15K09017).

\section{Availability of data and materials}

The datasets generated and/or analyzed during the current study are not publicly available due to confidentiality but are available from the corresponding author on reasonable request.

\section{Authors' contributions}

NI performed laboratory experiments with the help of AT, YG, KT, TK, TS, NY, OD, YS, AU, TN, KY, MM, HK, YN and YI, and prepared the manuscript. KY designed the present study and prepared the manuscript. All authors read and approved the final manuscript.

\section{Ethics approval and consent to participate}

The present study, including the collection and analytical methods, was approved by the Ethics Committees of the Kyoto Prefectural University of Medicine, and was conducted in accordance with the Declaration of Helsinki. Written informed consent was obtained from each patient for the use of their tissue samples in the present study.

\section{Consent for publication}

Written informed consent was obtained from each patient.

\section{Competing interests}

The authors have no conflicts of interest to disclose.

\section{References}

1. Torre LA, Bray F, Siegel RL, Ferlay J, Lortet-Tieulent J and Jemal A: Global cancer statistics, 2012. CA Cancer J Clin 65: 87-108, 2015.

2. El-Serag HB: Hepatocellular carcinoma. N Engl J Med 365: 1118-1127, 2011.

3. Ambros V: The functions of animal microRNAs. Nature 431: 350-355, 2004.

4. Bartel DP: MicroRNAs: Genomics, biogenesis, mechanism, and function. Cell 116: 281-297, 2004.

5. He L and Hannon GJ: MicroRNAs: Small RNAs with a big role in gene regulation. Nat Rev Genet 5: 522-531, 2004.

6. Calin GA, Dumitru CD, Shimizu M, Bichi R, Zupo S, Noch E, Aldler H, Rattan S, Keating M, Rai K, et al: Frequent deletions and down-regulation of micro-RNA genes miR15 and miR16 at 13q14 in chronic lymphocytic leukemia. Proc Natl Acad Sci USA 99: 15524-15529, 2002.

7. Lujambio A and Lowe SW: The microcosmos of cancer. Nature 482: 347-355, 2012.

8. Mao B and Wang G: MicroRNAs involved with hepatocellular carcinoma (Review). Oncol Rep 34: 2811-2820, 2015.

9. Mohammad RM, Muqbil I, Lowe L, Yedjou C, Hsu HY, Lin LT, Siegelin MD, Fimognari C, Kumar NB, Dou QP, et al: Broad targeting of resistance to apoptosis in cancer. Semin Cancer Biol 35 (Suppl): S78-S103, 2015.

10. Crawford ED, McNew JA, Nagata S and Wells J: Cell death. In: Molecular Biology of the Cell. 6th edition. Alberts B, Johnson A, Lewis J, Morgan D, Raff M, Roberts K and Walter P (eds). Garland Science, New York, pp1021-1034, 2015.

11. Soengas MS, Alarcón RM, Yoshida H, Giaccia AJ, Hakem R, Mak TW and Lowe SW: Apaf-1 and caspase-9 in p53-dependent apoptosis and tumor inhibition. Science 284: 156-159, 1999.

12. Xu D, He X, Chang Y, Xu C, Jiang X, Sun S and Lin J: Inhibition of miR-96 expression reduces cell proliferation and clonogenicity of HepG2 hepatoma cells. Oncol Rep 29: 653-661, 2013.

13. Leung WK, He M, Chan AW, Law PT and Wong N: Wnt/ $\beta$-catenin activates MiR-183/96/182 expression in hepatocellular carcinoma that promotes cell invasion. Cancer Lett 362: 97-105, 2015.

14. Guttilla IK and White BA: Coordinate regulation of FOXO1 by miR-27a, miR-96, and miR-182 in breast cancer cells. J Biol Chem 284: 23204-23216, 2009.

15. Fendler A, Jung M, Stephan C, Erbersdobler A, Jung K and Yousef GM: The antiapoptotic function of miR-96 in prostate cancer by inhibition of FOXO1. PLoS One 8: e80807, 2013. 
16. Myatt SS, Wang J, Monteiro LJ, Christian M, Ho KK, Fusi L, Dina RE, Brosens JJ, Ghaem-Maghami S and Lam EW: Definition of microRNAs that repress expression of the tumor suppressor gene FOXO1 in endometrial cancer. Cancer Res 70: 367-377, 2010

17. Yasui K, Konishi C, Gen Y, Endo M, Dohi O, Tomie A, Kitaichi T, Yamada N, Iwai N, Nishikawa T, et al: EVI1, a target gene for amplification at 3q26, antagonizes transforming growth factor- $\beta$-mediated growth inhibition in hepatocellular carcinoma. Cancer Sci 106: 929-937, 2015.

18. Zen K, Yasui K, Nakajima T, Zen Y, Zen K, Gen Y, Mitsuyoshi H, Minami M, Mitsufuji S, Tanaka S, et al: ERK5 is a target for gene amplification at $17 \mathrm{p} 11$ and promotes cell growth in hepatocellular carcinoma by regulating mitotic entry. Genes Chromosomes Cancer 48: 109-120, 2009.

19. Endo M, Yasui K, Zen Y, Gen Y, Zen K, Tsuji K, Dohi O, Mitsuyoshi H, Tanaka S, Taniwaki M, et al: Alterations of the SWI/SNF chromatin remodelling subunit-BRG1 and BRM in hepatocellular carcinoma. Liver Int 33: 105-117, 2013.

20. Pineau P, Volinia S, McJunkin K, Marchio A, Battiston C Terris B, Mazzaferro V, Lowe SW, Croce CM and Dejean A: miR-221 overexpression contributes to liver tumorigenesis. Proc Natl Acad Sci USA 107: 264-269, 2010.

21. Ress AL, Stiegelbauer V, Winter E, Schwarzenbacher D, Kiesslich T, Lax S, Jahn S, Deutsch A, Bauernhofer T, Ling H, et al: MiR-96-5p influences cellular growth and is associated with poor survival in colorectal cancer patients. Mol Carcinog 54: $1442-1450,2015$.

22. Schaefer A, Jung M, Mollenkopf HJ, Wagner I, Stephan C, Jentzmik F, Miller K, Lein M, Kristiansen G and Jung K: Diagnostic and prognostic implications of microRNA profiling in prostate carcinoma. Int J Cancer 126: 1166-1176, 2010.

23. Han Y, Chen J, Zhao X, Liang C, Wang Y, Sun L, Jiang Z, Zhang Z, Yang R, Chen J, et al: MicroRNA expression signatures of bladder cancer revealed by deep sequencing. PLoS One 6 : e18286, 2011.

24. Li C, Yin Y, Liu X, Xi X, Xue W and Qu Y: Non-small cell lung cancer associated microRNA expression signature: Integrated bioinformatics analysis, validation and clinical significance. Oncotarget 8: 24564-24578, 2017.

25. Siu MK, Tsai YC, Chang YS, Yin JJ, Suau F, Chen WY and Liu YN: Transforming growth factor- $\beta$ promotes prostate bone metastasis through induction of microRNA-96 and activation of the mTOR pathway. Oncogene 34: 4767-4776, 2015.
26. Ma Y, Yang HZ, Dong BJ, Zou HB, Zhou Y, Kong XM and Huang YR: Biphasic regulation of autophagy by miR-96 in prostate cancer cells under hypoxia. Oncotarget 5: 9169-9182, 2014.

27. Jeong HJ, Park SY, Yang WM and Lee W: The induction of miR-96 by mitochondrial dysfunction causes impaired glycogen synthesis through translational repression of IRS-1 in SK-Hep1 cells. Biochem Biophys Res Commun 434: 503-508, 2013.

28. Calnan DR and Brunet A: The FoxO code. Oncogene 27: 2276-2288, 2008.

29. Dong XY, Chen C, Sun X, Guo P, Vessella RL, Wang RX, Chung LW, Zhou W and Dong JT: FOXO1A is a candidate for the 13 q14 tumor suppressor gene inhibiting androgen receptor signaling in prostate cancer. Cancer Res 66: 6998-7006, 2006.

30. Lin H, Dai T, Xiong H, Zhao X, Chen X, Yu C, Li J, Wang X and Song L: Unregulated miR-96 induces cell proliferation in human breast cancer by downregulating transcriptional factor FOXO3a. PLoS One 5: e15797, 2010.

31. Jalvy-Delvaille S, Maurel M, Majo V, Pierre N, Chabas S, Combe C, Rosenbaum J, Sagliocco F and Grosset CF: Molecular basis of differential target regulation by miR-96 and miR-182: The Glypican-3 as a model. Nucleic Acids Res 40: 1356-1365, 2012.

32. Zhang J, Kong X, Li J, Luo Q, Li X, Shen L, Chen L and Fang L: miR-96 promotes tumor proliferation and invasion by targeting RECK in breast cancer. Oncol Rep 31: 1357-1363, 2014.

33. Xu L, Zhong J, Guo B, Zhu Q, Liang H, Wen N, Yun W and Zhang L: miR-96 promotes the growth of prostate carcinoma cells by suppressing MTSS1. Tumour Biol 37: 12023-12032, 2016.

34. Hong Y, Liang H, Uzair-Ur-Rehman, Wang Y, Zhang W, Zhou Y, Chen S, Yu M, Cui S, Liu M, et al: miR-96 promotes cell proliferation, migration and invasion by targeting PTPN9 in breast cancer. Sci Rep 6: 37421, 2016.

35. Velagapudi SP, Gallo SM and Disney MD: Sequence-based design of bioactive small molecules that target precursor microRNAs. Nat Chem Biol 10: 291-297, 2014.

36. Velagapudi SP, Cameron MD, Haga CL, Rosenberg LH, Lafitte M, Duckett DR, Phinney DG and Disney MD: Design of a small molecule against an oncogenic noncoding RNA. Proc Natl Acad Sci USA 113: 5898-5903, 2016. 